\title{
Durch Absatzschuhe auf falsche Sitztoiletten Ein Denkanstoß
}

Der absatzbetonte Schuh hat nicht nur das Gehen des Menschen verändert, sondern auch die tiefe Hocke erschwert. Der zivilisierte Toilettengang auf dem erhöhten „Sitzthron“ wurde „erkauft" durch eine blutdrucksteigernde Pressatmung und ihre Folgen auf die menschliche Gesundheit.

Naturvölker sind Barfuß- oder Sandalengeher, allein hierdurch wird der Fuß natürlich über Ferse und Vorfuß abgerollt, die Waden-/Achillessehnen sind optimal gedehnt, sodass auch in der Hocke der Fuß stabil in ganzer Länge am Boden haften kann. Mit Ausgang des Mittelalters haben wir dieses natürliche Verhalten geändert, der Absatzschuh machte Karriere, man konnte sich auf diese Weise besser in Position bringen (Abb. 1). Der Gang veränderte sich in das absatzbetonte Vorfußgehen, bei dem die Beine nur noch einseitig frontal ausgerichtet sind, der energiefördernde Gegenschwung aber auf der Strecke blieb.

\section{Zivilisierter ,Stuhlgang“ \\ mit negativen Folgen}

Der absatzbetonte Schuh hat aber nicht nur das Gehen des Menschen verändert, sondern auch die Bodenarbeiten er-
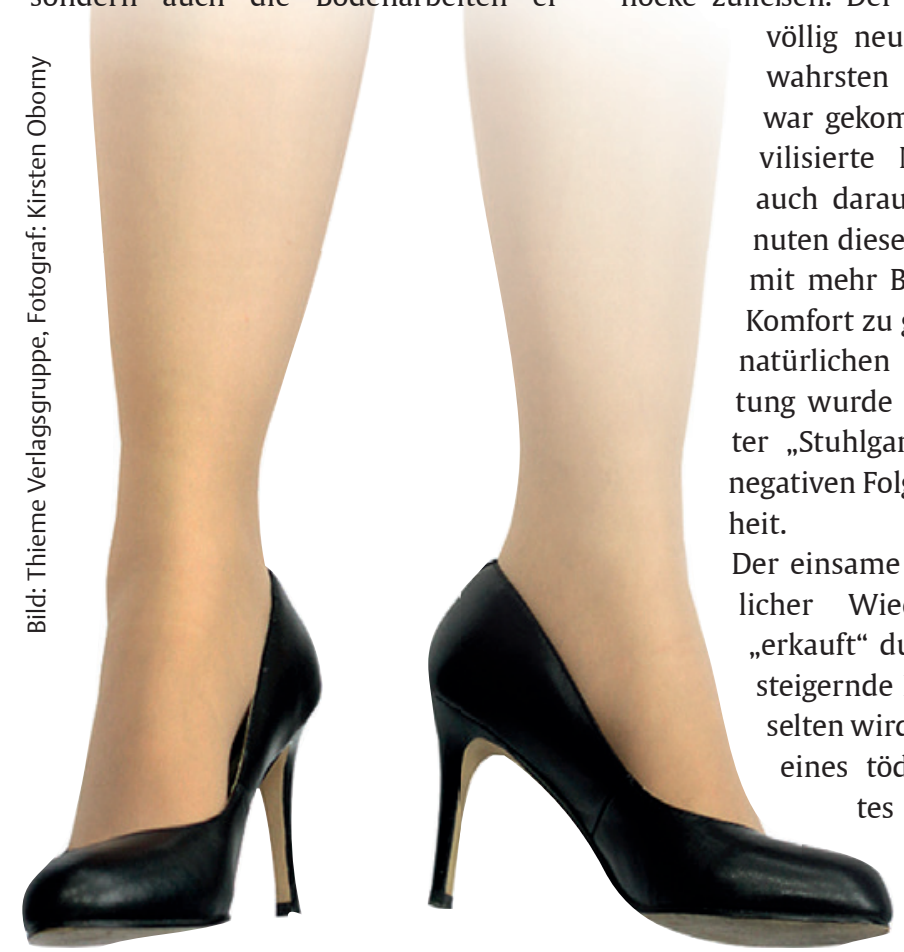

Abb. 1 Hohe Absatzschuhe provozieren Waden-und Achillessehnenverkürzungen, die die natürliche Entspannungshocke nicht mehr zulassen. schwert. Denn die Absatzerhöhung geht einher mit einer zunehmenden Verkürzung der Waden- und Achillessehen, sodass in der tiefen Hockstellung nicht mehr beide Füße fest mit den Fersen am Boden verankert werden können (Abb. 2). In tiefer Arbeitshocke ist man also gezwungen, die Fersen anzuheben, auf den Vorfüßen zu balancieren und meniskusbelastend die Kniegelenke nach außen zu drehen. Durch die erzwungene Außenrotation unter Körperbelastung degenerieren die Innenmenisken derart, dass häufig schon ein Bagatellunfall im Sinne der einfachen Knieverdrehung genügt, einen Meniskusriss hervorzurufen.

Aber auch die tägliche Darmentleerung mehr oder weniger in „freier Wildbahn“ erwies sich plötzlich als problematisch, weil die verkürzten Waden- und Achillessehnen nur noch eine mühevolle Krampfhocke zuließen. Der Moment für einen völlig neuen „Stuhlgang“ im wahrsten Sinne des Wortes war gekommen, denn der zivilisierte Mensch war jetzt auch darauf bedacht, die Minuten dieser Abgeschiedenheit mit mehr Bequemlichkeit und Komfort zu gestalten. Aus einer atürlichen täglichen Verrichtung wurde ein ganz bestimmter „Stuhlgang“, allerdings mit negativen Folgen für die Gesundheit.

Der einsame „Sitzthron“ in tägicher Wiederholung wurde erkauft" durch die blutdruckeigernde Pressatmung. Nicht elten wird der Notarzt wegen ichen Herzinfarkes auf einem stillen Örtchen gerufen. In der Sportmedizin warnt man schon lange vor der Pressatmung bei einfachen
Liegestützen und die negativen Folgen auf die menschliche Gesundheit.

\section{Probleme der Pressatmung beim Valsalva-Manöver}

Jede Form der Pressatmung stellt eine Sauerstoffkrise im Körper dar. Durch unsere falschen Toilettengewohnheiten findet dieses Manöver bis zum 50. Lebensjahr praktisch bei jedem von uns 73000 Mal statt, wenn auf der Sitztoilette täglich 4-mal pro Sitzung das Valsalva-Manöver praktiziert wird (Tab. 1).

Mit der Einführung der heutigen Sitztoiletten Mitte des 19. Jahrhunderts war auch ein Anstieg der Blinddarmentzündungen zu verzeichnen, die bis dahin praktisch unbekannt waren. 1886 wurde erstmalig die Diagnose „Appendizitis“ durch Prof. Reginald Heber Fritz von der Harvard Universität gestellt und er war auch der Erste, der die operative Entfernung des entzündeten Dickdarmfortsatzes empfahl. Mit einer grundsätzlichen unnatürlichen Verhaltensänderung begann also der Anstieg von Blinddarmentzündungen als eine der ersten Zivilisationserkrankungen. Was macht der Mensch auf der Sitztoilette anders, als der Naturmensch in "freier Wildbahn“? Auf der Sitztoilette sind, im Vergleich zur freien Hocke, die Hüftgelenke um $90^{\circ}$ gebeugt, sodass die Oberschenkel weit vom Körper entfernt eingestellt sind. In der natürlichen Hocksitzhaltung dagegen sind die Hüftgelenke maximal gebeugt, sodass beide Oberschenkel intensiv gegen die Bauchwand gepresst werden, dabei drückt der rechte Oberschenkel gegen das Coecum (Grimmdarm) als Teil des aufsteigenden Dickdarms und der linke Oberschenkel gegen den absteigenden Dickdarm mit der Sigmaschlinge. Allerdings werden im vorgerückten Alter gute Ernährung und Fehlbelastungen im Knie Veränderungen hinterlassen haben, sodass die freie Hocke nicht mehr praktizierbar ist. Hilfreich ist dann ein Hocker vor der Toilette, der die Oberschenkel so weit anhebt, dass der Oberschenkeldruck mit gebeugtem Oberkörper und durch den Zug mit den Armen möglich ist.

In der unnatürlichen Sitzhaltung auf der modernen Wassertoilette fällt die äuße- 


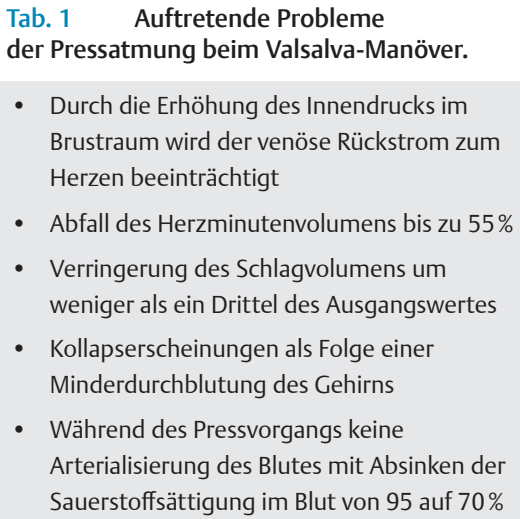

Tab. 1 Auftretende Probleme

der Pressatmung beim Valsalva-Manöver.

- Durch die Erhöhung des Innendrucks im Brustraum wird der venöse Rückstrom zum Herzen beeinträchtigt

- Abfall des Herzminutenvolumens bis zu 55\%

- Verringerung des Schlagvolumens um weniger als ein Drittel des Ausgangswertes

- Kollapserscheinungen als Folge einer Minderdurchblutung des Gehirns

- Während des Pressvorgangs keine Arterialisierung des Blutes mit Absinken der Sauerstoffsättigung im Blut von 95 auf $70 \%$

re Kompression durch die Oberschenkel vollständig weg. Der tägliche Toilettengang wird so zu einem Drücken aus dem Kopf heraus, mit gepresstem Atem wird das Zwerchfell nach unten in den Bauchraum gedrückt, nur so kann die Darmpassage beschleunigt werden Die negativen Folgen aus diesem Verhalten sind leicht nachvollziehbar, denn dieses Valsalva-Manöver fördert nicht die Stuhlentleerung, sie erhöht vielmehr den Druck in die Gegenrichtung.

Am stärksten wird in westlichen Ländern das Valsalva-Manöver bei der Geburt praktiziert, dabei wird allerdings im Liegen oder in Halb-Sitzposition der Geburtskanal um 20-30\% eingeengt im Vergleich zur natürlichen Hocksitzhaltung. Diesen Widerstand gilt es zu überwinden, der Kopf des Babys wird in den verengten Geburtskanal hineingepresst, oft unterstützt durch Zangen oder Saugglocken, dabei müssen vielfach Verletzungen teuer „erkauft“ werden.

Erkrankungen von Dünn- und Dickdarm Die erste Druckwelle von oben herab trifft auf den Grimmdarm (Coecum) mit dem Blinddarm. Stuhlanteile werden so über einen Kanal ins Lumen gedrückt und können jederzeit eine Blinddarmentzündung (Appendizitis) in Erscheinung treten lassen. Eine weitere Druckwelle über das Pressen kann die Ileocoecalklappe zum Dünndarm öffnen, ein Ventil, das normalerweise in dieser Situation geschlossen ist. Damit fließen Stuhlanteile in den Dünndarm zurück, die hier zu Entzündungen führen können - wahrscheinlich die wesentliche Ursache für die Entstehung der Ileitis terminali (Dünndarmentzündung) und des Morbus Crohn.

Ferner kann die Druckerhöhung durch Pressatmung die Entstehung von Divertikeln des Dickdarms bewirken, beson- ders im Bereich des Sigmas. Durch den fehlenden Oberschenkeldruck bei der Darmentleerung erhöht sich neben dem Druck auch der Knickwinkel im Sigma, sodass die Ausscheidungsmenge sich auf den geringen Darmanteil unterhalb der Abknickung beschränkt. Gleichzeitig presst das Valsalva-Manöver Stuhlreste in die Divertikel, die häufig entzündliche Veränderungen provozieren. Eine solche Divertikulitis ist vergleichbar mit der Appendizitis, wobei auf lange Sicht betrachtet jedes Divertikel auch bösartig entarten kann.

Auch die Tendenz zu Leistenbrüchen (direkte und indirekte Hernien) ist nicht allein anlagebedingt, sondern auch im Zusammenhang mit einem erhöhten Pressdruck im Bauchraum zu sehen. Dies gilt auch für die Zwerchfellhernie in $\mathrm{Zu}$ sammenhang mit der Refluxösophagitis (GERD - Gastroesophagal Reflux Disease), bei der Magensäure in die Speiseröhre gelangen kann, eine klare Präkanzerose. Im American Journal of Clinical Nutrition hat bereits 1981 D. Burkitt auf der Basis des gemessenen erhöhten Bauchhöhlendrucks bei der Stuhlentleerung den Zusammenhang mit westlichen Sitztoiletten und der Entstehung von Hiatushernien nachgewiesen. In radiologischen Untersuchungen ist dokumentiert, dass Hiatushernien bei $20 \%$ der nordamerikanischen Bevölkerung auftreten, was auch für die wirtschaftlich entwickelten Gebiete in Westeuropa gilt. In umfangreichen radiologischen Kontrastuntersuchungen zeigten sich in Afrika weit weniger Hiatusfälle, in Kenia 1 Person auf 1000, in Nigeria 4 von 1030, in Tansania 1 von 700 [1].

Auch der Dickdarmkrebs steht mit dem Hang zur Verstopfung in Zusammenhang, ein typisches Zeichen im Stresszeitalter. Der knapp 2 Meter lange Dickdarmschlauch befördert seinen Inhalt durch Wandpulsationen in Richtung Ausgang, wobei ständig Wasser rückresorbiert wird. Ist die Passage durch unnatürliches Verhalten gestört, so kann sich der Stuhl zementartig durch die Austrocknung verhärten und an den Darmwänden festsetzen. Allein in den USA erkranken im Jahr ca. 150000 Menschen an Darmkrebs. Exzessives Pressen bei der Stuhlentleerung provoziert zudem Hämorrhoiden, hie- runter leiden in westlichen Ländern bis zu 50\% der Menschen über 40 Jahren. Auch die üblichen Geburtsvorgänge in westlichen Ländern werden von überaus starken Valsalva-Manövern beherrscht, sodass es nicht überrascht, wenn viele Mütter sich nach einer Geburt jahrelang mit Hämorrhoiden herumplagen müssen.

\section{Erkrankungen des Beckenbodens}

Die Muskel-Sehnenplatte des Beckenbodens wird wesentlich vom Pudendusnerv gesteuert, der neben motorischen auch über parasympathische Fasern verfügt. Dieser Nerv steuert nachhaltig die Funktionen der Gebärmutter mit den Adnexen (Eierstöcke und Eileiter), die Prostata, aber auch die Stabilität des gesamten Beckenbodens. Durch das wiederholte Valsalva-Manöver auf den herkömmlichen Toiletten kommt es neben der Insuffizienz des Beckenbodens auch zu einer Druckschädigung des Pudendusnerven. Damit verlieren insbesondere die weiblichen Beckenorgane ihr „Gedächtnis“, Hormonstörungen treten in den Vordergrund, wie sie aktuell in westlichen Ländern an Bedeutung gewinnen. Die Endometriose, eine schmerzhafte, chronische Erkrankung bei jungen Frauen ist die Folge eines falschen Zellverhaltens, wenn Zellen ihre nervliche Steuerung zum Gehirn verlieren. Diese gestörten Zellen erreichen nicht die Gebärmutter, sie wandern $\mathrm{ab}$ und siedeln sich in anderen Organen an. Auf diese Weise können auch Gebärmuttermyome oder gar Krebs entstehen. An der Endometriose leiden allein in den USA und Kanada 51\%2 Millionen Frauen und Mädchen und weitere Millionen weltweit, belegt durch Zahlen der „Endometriosis Association“. Allein in den USA werden pro Jahr mehr als 600000 Gebärmutterentfernungen durchgeführt, das sind ein Drittel aller 60-Jährigen [2]. Die Harnblaseninkontinenz und andere Vorfälle betreffen Frauen in besonderer Weise, weil neben der Schädigung des

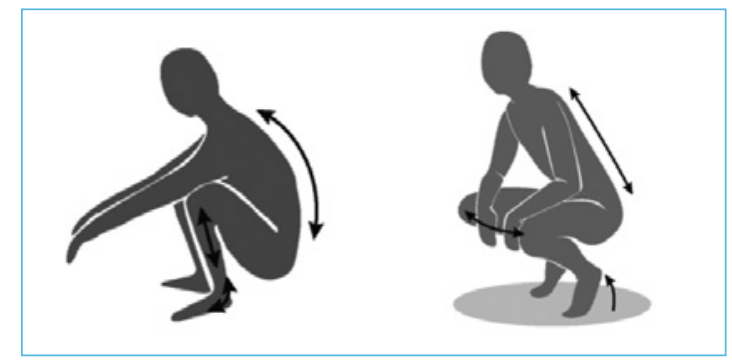

Abb. 2 Tiefe Hocke. a) Natürliche Entspannungshocke, b) Europäische „Krampfhocke“. 
Abb. 3 Einübung der natürlichen Entspannungshocke durch Tür- und Partnerunterstützung.

Beckenbodens durch das wiederholte Valsalva-Manöver auch die natürlichen weiblichen Lücken des Beckenbodens berücksichtigt werden müssen, hervorgerufen durch den Vaginalkanal. Die ständigen Pressvorgänge auf den westlichen Toiletten bewirken Beckenorganvorfälle: Gebärmutter-, Harnblasen(Zystozele), Mastdarm- (Rektozele) und Darmvorfälle (Enterozele).

Die Prostata und Harnblase werden ebenso vom Pudendusnerv kontrolliert, der aus dem Kreuzbein in die Beckenregion und parallel zur Wirbelsäule in die Dammregion gelangt. Beim druckgeschädigten Nerven durch wiederholtes Pressen wird die nervöse Steuerung der Prostata unterbrochen, was unweigerlich mit Funktionsstörungen verbunden ist. Zu Beginn zeigt sich in der Regel eine Vergrößerung der Prostata, es folgen Entzündungen bis zum weit verbreiteten Prostatakrebs im vorgerückten Alter. Der Pudendusnerv verläuft durch den Beckenboden, diese Muskelplatte kann das tägliche Valsalva-Manöver dauerhaft nicht kompensieren, sie wird überdehnt. Dabei sind speziell die Nerven betroffen, weil sie über kein Dehnungsvermögen verfügen, eine Überdehnung um 12 \% zerstört bereits das Nervengewebe. Auf diese Weise verliert der Pudendusnerv seine nervöse Kontrolle über die Prostata, die Harnblase und über die weiblichen Beckenorgane.

\section{Ein Lösungsansatz:} die "Saigonhocke“

Die „Saigonhocke“ ist eine tiefe, natürliche Entspannungshocke, meine langjährige Erfahrung aus dem Vietnamkrieg als Chirurg. Sie bietet ein Musterbeispiel optimaler Entspannung: einmal für den Rücken mit seiner Muskulatur bei gleichzeitiger Erweiterung des Spinalkanals, zum anderen ist sie eine Lösung gegen Waden- und Achillessehnenstress. Um die freie „Saigonhocke“ auf die Beine zu bringen, hilft zunächst ein einfacher Schemel. Erlernt wird die Hocke vor einer geöffneten Tür, man hält sich mit den Händen an den Türgriffen und geht in die Hocke, die Füße stehen parallel, die Fersen fest am Boden, das Becken wird maximal abgesenkt, um den Rücken zu runden. Spannt man jetzt mehrmals nacheinander kurz die Waden an, werden auch hierdurch die Achillessehnen elastischer (Abb. 3). Beim Partnerstretching halten Sie mit gekrallten Händen den Partner, gehen mit gestreckten Armen in die tiefe Hocke und verlagern die Wirbelsäule nach hinten. Jetzt schaukeln Sie gemeinsam vor und zurück und intensivieren so die Entspannung des Rückens.

\section{Literatur}

1 Burkitt DP. Hiatus hernia: is it preventable? Am J Clinl Nutr 1981; 34: 428-431

2 Mclntosh $\mathrm{L}$. The role of the nurse in the use of vaginal pessaries to treat pelvic organ prolapse and/or urinary incontinence: a literature review. Urologic Nursing 2005; 25 : $41-48$

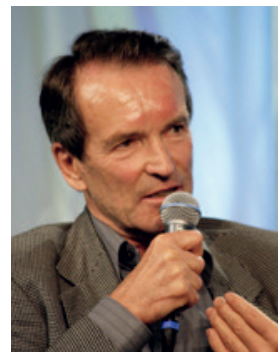

Korrespondenz

Prof. Dr. med. Gerd Schnack

Allensbacher

Präventionszentrum

Hirschweg 15

78476 Allensbach/

Bodensee

Tel.: 07533/997676

www.praeventions

zentrum.com 